\title{
银催化炔烃与有机高价碘(III)试剂的立体选择性加成反应
}

\author{
陈新宇 ${ }^{a}$ 刘雪艳 $b$ 马 方 ${ }^{a}$ 洪先芳 ${ }^{a}$ 李洪基 $*, a$ \\ ( ${ }^{a}$ 淮北师范大学化学与材料科学学院 教育部绿色和精准合成化学及应用重点实验室 安徽淮北 235000) \\ $\left({ }^{b}\right.$ 蚌埠产品质量监督检验研究院 安徽蚌埠 233040)
}

\begin{abstract}
摘要 报道了一种银催化功能化炔烃与有机高价碘(III)试剂的立体选择性加成反应. 该反应体系底物适用范围广，可 以中等到优秀的收率得到烯基酯类化合物. 分子内的控制实验证实, 有机高价碘试剂分子中的芳基显著促进与其相连 的 $\mathrm{I}-\mathrm{O}$ 键优先断裂, 进而参与后续的立体选择性加成反应

关键词＼cjkstart银催化; 炔烃; 高价碘(III)试剂; 立体选择性加成
\end{abstract}

\section{Silver-Catalyzed Stereoselective Addition of Organic Hypervalent lodine(III) Reagents to Alkynes}

\author{
Chen, Xinyu ${ }^{a} \quad$ Liu, Xueyan ${ }^{b} \quad$ Ma, Fang ${ }^{a} \quad$ Hong, Xianfang $^{a} \quad$ Li, Hongji ${ }^{*, a}$ \\ $\left({ }^{a}\right.$ Key Laboratory of Green and Precise Synthetic Chemistry and Applications, Ministry of Education, \\ School of Chemistry and Materials Science, Huaibei Normal University, Huaibei, Anhui 235000) \\ ( ${ }^{b}$ Bengbu Product Quality and Inspection Institute, Bengbu, Anhui 233040)
}

\begin{abstract}
A silver-catalyzed stereoselective addition reaction of functionalized alkynes with organic hypervalent iodine(III) reagents as nucleophiles is reported, providing an approach to vinyl esters in high yields with excellent group tolerance. Intramolecular experiment demonstrates that the presence of aryl group considerably affects the cleavage of $\mathrm{I}-\mathrm{O}$ bond within hypervalent iodine(III) reagents, which is also responsible for the high stereoeselectivity observed in this catalytic system.

Keywords silver catalysis; alkyne; hypervalent(III) iodine reagent; stereoselective addition
\end{abstract}

\section{Introduction}

The functionalization of alkynes represents an extremely powerful tool for the construction of chemical bonds, which has widespread application in organic synthesis. ${ }^{[1]}$ Alkynes usually are used as feedstock to afford a series of valuable commodity chemicals, such as acetaldehydes, ketones, vinyl esters and some unique compounds that are not easy to obtain by conventional synthetic methods. ${ }^{[2]}$ Particularly, in the case of functionalized alkynes, a number of vinyl esters can be prepared from the addition reaction of carbon-carbon triple bond, in which the pursuit of good selectivity remains a significant challenge. In recent years, considerable effort has been devoted to explore the versatile additions of alkynes toward achieving high selectivity. ${ }^{[3]}$ For example, Jiang's group ${ }^{[4]}$ has realized the difunctionalization of terminal alkynes by silver catalysis, generating a variety of 1-halo-1-alkenes in good yields and selectivity (Scheme 1a). Hou and coworkers ${ }^{[5]}$ have established the halogenation of internal alkynes using quaternary ammonium salt as mediators. Then it is found that both phenols and 4-hydroxyl coumarins are effective $O$-containing nucleophiles, which can enable the stereoselective addition of alkynes (Schemes $1 b$ and $1 \mathrm{c}) .{ }^{[6]}$ Very recently, $\mathrm{Xu}$ et al. $^{[7]}$ reported an efficient addition of sulfonic acids to haloalkynes, in which the reactivity of sulfonic acids can be tuned by varying the hydrogen bonding cluster (Scheme 1, d). Moreover, it should be noted that the direct addition of carboxyl acid to propiolates also proceeds well under palladium catalysis (Scheme 1, e) ${ }^{[8]}$ Although the reported strategies have been well developed for the alkyne addition, most of which suffer from poor selectivity or critical reaction conditions. Hence, the de-

\footnotetext{
* Corresponding author. E-mail: hongjili@chnu.edu.cn

Received April 27, 2020; revised June 23, 2020; published online June 30, 2020

Dedicated to Professor Henry N. C. Wong on the occasion of his 70th birthday.

Project supported by the National Natural Science Foundation of China (No. 21772061) and the Natural Science Foundation for the Higher Education Institutions of Anhui Province (No. KJ2017A388).

国家自然科学基金(No. 21772061)和安徽省高校自然科学基金(No. KJ2017A388)资助项目.
} 


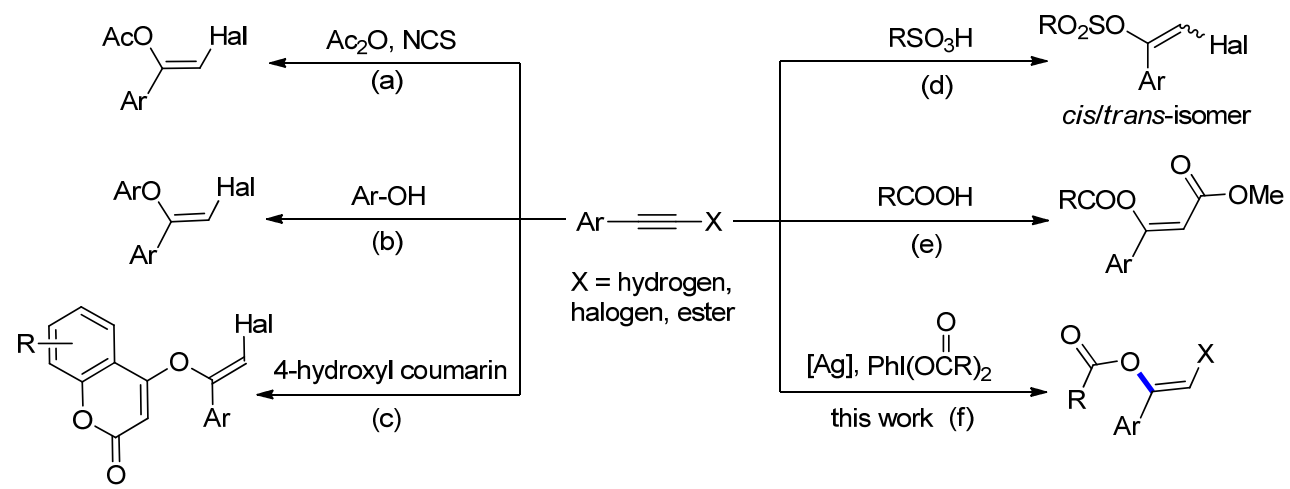

Scheme 1 Stereoselective addition reactions of alkynes with $O$-containing nucleophiles

velopment of an efficient and alternative methodology for the alkyne addition with good selectivity is highly desirable.

Organic hypervalent iodine reagents (HIRs) have gained increasing interest as coupling partners, oxidants and both of them in organic synthesis, mainly due to their selective, versatile and environment-benign properties. ${ }^{[9]}$ Several typical organoiodine(III) reagents have been successfully employed for the construction of organic molecules. ${ }^{[9-10]}$ Among them, [bis(acyloxy)iodo]arenes (DIB) and [hydroxy(tosyloxy)iodo]benzene (HTIB, Koser's reagent), belong to one such reagent that has been frequently explored in organic transformations. ${ }^{[10]}$ For example, we recently have utilized the organoiodine(III) reagents to realize the selective $\mathrm{C}\left(\mathrm{sp}^{3}\right)-\mathrm{H}$ acetoxylation of $2 H$-aziridines ${ }^{[11 \mathrm{c}]}$ and radical cyclization of unsaturated oximes, ${ }^{[11 \mathrm{~d}]}$ respectively. During the course of the investigation, it was found that the cleavage of $\mathrm{I}-\mathrm{O}$ bond counld be facilitated by iron(II/III) salts. We then postulated the possible cleavage model of $\mathrm{I}-\mathrm{O}$ bond mediated by other transition metals, such as silver salts. In this contribution, we will report an example of silver-catalyzed $\mathrm{I}-\mathrm{O}$ cleavage within HIRs derived from DIB and the corresponding addition to functionalized alkynes (Scheme 1f).

\section{Results and discussion}

Based on previous work, ${ }^{[11]}$ we commenced this study with the reaction of (bromoethynyl)benzene 1a with hypervalent iodine reagent 2a to evaluate the feasibility of the process, and the results are summarized in Table 1. Initially, $\mathrm{Ag}_{2} \mathrm{O}$ was selected as a catalyst for the catalytic system. ${ }^{[6 b]}$ The model reaction proceeded slightly in $\mathrm{CH}_{3} \mathrm{CN}$ at $90{ }^{\circ} \mathrm{C}$ for $6 \mathrm{~h}$, and the desired product $3 \mathrm{a}$ was generated in $21 \%$ isolated yield (Entry 1). It shoud be noted that the NOSEY spectra of $\mathbf{3 a}$ further supported the observed stereoslectivity. Other silver salts, such as AgOAc, AgOTf and $\mathrm{AgNO}_{3}$ were then tried for the addition reaction of $\mathbf{1 a}$, but failed to give the product 3a (Entries 2 4). Gratifyingly, it was found that $\mathrm{Ag}_{2} \mathrm{CO}_{3}$ exhibited high efficiency in this reaction system, affording 3a in $86 \%$ yield (Entry 5). Instead of $\mathrm{Ag}_{2} \mathrm{CO}_{3}$, the use of some other catalysts including $\mathrm{CuO}$ and $\mathrm{Cu}_{2} \mathrm{O}, \mathrm{Fe}(\mathrm{acac})_{2}, \mathrm{Fe}(\mathrm{acac})_{3}$ and $\mathrm{Pd}(\mathrm{OAc})_{2}$ did not promote this addition process (Entries $6 \sim 10$ ). No

Table 1 Optimization of reaction conditions ${ }^{a}$

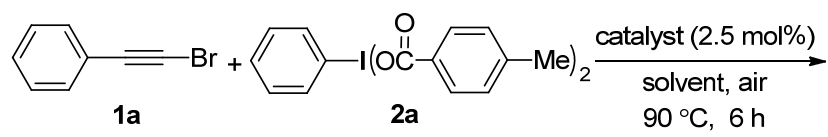

1a<smiles>Cc1ccc(C(=O)O/C(=C\Br)c2ccccc2)cc1</smiles>

\begin{tabular}{|c|c|c|c|}
\hline Entry & Catalyst & Solvent & Yield $^{b} / \%$ \\
\hline 1 & $\mathrm{Ag}_{2} \mathrm{O}$ & $\mathrm{CH}_{3} \mathrm{CN}$ & 21 \\
\hline 2 & $\mathrm{AgOAc}$ & $\mathrm{CH}_{3} \mathrm{CN}$ & 0 \\
\hline 3 & AgOTf & $\mathrm{CH}_{3} \mathrm{CN}$ & 0 \\
\hline 4 & $\mathrm{AgNO}_{3}$ & $\mathrm{CH}_{3} \mathrm{CN}$ & 0 \\
\hline 5 & $\mathrm{Ag}_{2} \mathrm{CO}_{3}$ & $\mathrm{CH}_{3} \mathrm{CN}$ & 86 \\
\hline 6 & $\mathrm{CuO}$ & $\mathrm{CH}_{3} \mathrm{CN}$ & 0 \\
\hline 7 & $\mathrm{Cu}_{2} \mathrm{O}$ & $\mathrm{CH}_{3} \mathrm{CN}$ & Trace \\
\hline 8 & $\mathrm{Fe}(\mathrm{acac})_{2}$ & $\mathrm{CH}_{3} \mathrm{CN}$ & 0 \\
\hline 9 & $\mathrm{Fe}(\mathrm{acac})_{3}$ & $\mathrm{CH}_{3} \mathrm{CN}$ & 0 \\
\hline 10 & $\mathrm{Pd}(\mathrm{OAc})_{2}$ & $\mathrm{CH}_{3} \mathrm{CN}$ & 0 \\
\hline 11 & - & $\mathrm{CH}_{3} \mathrm{CN}$ & 0 \\
\hline 12 & $\mathrm{Ag}_{2} \mathrm{CO}_{3}$ & $\mathrm{EtOH}$ & 15 \\
\hline 13 & $\mathrm{Ag}_{2} \mathrm{CO}_{3}$ & $\mathrm{AcOH}$ & 22 \\
\hline 14 & $\mathrm{Ag}_{2} \mathrm{CO}_{3}$ & THF & Trace \\
\hline 15 & $\mathrm{Ag}_{2} \mathrm{CO}_{3}$ & $\mathrm{DMF}$ & n.r. \\
\hline 16 & $\mathrm{Ag}_{2} \mathrm{CO}_{3}$ & DMSO & 17 \\
\hline 17 & $\mathrm{Ag}_{2} \mathrm{CO}_{3}$ & 1,4-Dioxane & Trace \\
\hline 18 & $\mathrm{Ag}_{2} \mathrm{CO}_{3}$ & $\mathrm{PhMe}$ & 0 \\
\hline 19 & $\mathrm{Ag}_{2} \mathrm{CO}_{3}$ & $\mathrm{CH}_{3} \mathrm{CN}$ & $65^{c}$ \\
\hline 20 & $\mathrm{Ag}_{2} \mathrm{CO}_{3}$ & $\mathrm{CH}_{3} \mathrm{CN}$ & $83^{d}$ \\
\hline 21 & $\mathrm{Ag}_{2} \mathrm{CO}_{3}$ & $\mathrm{CH}_{3} \mathrm{CN}$ & $72^{e}$ \\
\hline 22 & $\mathrm{Ag}_{2} \mathrm{CO}_{3}$ & $\mathrm{CH}_{3} \mathrm{CN}$ & $58^{f}$ \\
\hline 23 & $\mathrm{Ag}_{2} \mathrm{CO}_{3}$ & $\mathrm{CH}_{3} \mathrm{CN}$ & $78^{g}$ \\
\hline 24 & $\mathrm{Ag}_{2} \mathrm{CO}_{3}$ & $\mathrm{CH}_{3} \mathrm{CN}$ & $64^{h}$ \\
\hline
\end{tabular}

${ }^{a}$ Reaction conditions: 1a $(0.2 \mathrm{mmol}), \mathbf{2 a}(0.2 \mathrm{mmol})$ and catalyst $(2.5 \mathrm{~mol} \%)$ in $\mathrm{CH}_{3} \mathrm{CN}(1 \mathrm{~mL})$ at $90{ }^{\circ} \mathrm{C}$ under ambient air for $6 \mathrm{~h} .{ }^{b}$ Isolated yield.

${ }^{c} \mathrm{Ag}_{2} \mathrm{CO}_{3}(1 \mathrm{~mol} \%) .{ }^{d} \mathrm{Ag}_{2} \mathrm{CO}_{3}(5 \mathrm{~mol} \%) .{ }^{e} 80{ }^{\circ} \mathrm{C} .{ }^{f} 100{ }^{\circ} \mathrm{C} .{ }^{g} 8 \mathrm{~h} .{ }^{h} 5 \mathrm{~h}$.

product 3a was isolated in absence of $\mathrm{Ag}_{2} \mathrm{CO}_{3}$ (Entry 11). Following, it was found that the reaction of 1a with 2a was considerably affected by the reaction medium. For instance, using ethanol or acetic acid as a solvent did not remarkably accelerate the addition process, and only $15 \%$ 
and $22 \%$ yields were achieved under the above conditions, respectively (Entries 12 and 13). Moreover, some other polar solvents were also examined in the model reaction, and resulted into inferior yields (Entries 13 17). The reaction did proceed in toluene (Entry 18). Finally, the evaluation of reaction time, temperature and catalyst loading gave the optimal reaction conditions for the addition reaction of $\mathbf{1 a}$ with $\mathbf{2 a}$. The experiment results indicated that longer reaction time and higher temperature would lead to partial decomposition of 3a with lower isolated yield (Entries 19 24).

With the optimal conditions in hand, we next investigated the applicability of this method across a series of bromoalkynes, and the result is listed in Table 2. The bromoalkynes bearing aryl group could undergo the reaction with 2 a to deliver the related products in good yields. Notably, the para-substitued substrates with an electrondonating group such as $\mathrm{Me},{ }^{n} \mathrm{Pr},{ }^{n} \mathrm{Bu},{ }^{n} \mathrm{Pent}, \mathrm{MeO}$ and EtO, were well tolerated under the standard conditions $(\mathbf{3 b} \sim$ 3g). The incorporation of $\mathrm{F}$ and $\mathrm{Br}$ into the para position did not affect the silver-catalyzed system, generating the desired product in $89 \%$ (3h) and $86 \%$ (3i) yields, respectively. We then found that the bromoalkynes with electron- deficient groups including $\mathrm{CN}, \mathrm{NO}_{2}$ and $\mathrm{CF}_{3}$ afforded the products in excellent yields $(\mathbf{3} \mathbf{j} \sim \mathbf{3 l})$. It was found that 4-(bromoethynyl)-1,1'-biphenyl could react with $\mathbf{2 a}$ to give $76 \%$ yield of $3 \mathrm{~m}$. The use of meta-substituted bromoalkynes gave the according products in comparable yields, indicating weak steric hindrance exists in this process (3n and 3o). Introducing thienyl group into bromoalkynes still generated the desired products, albeit with moderate yields $(\mathbf{3 p} \sim \mathbf{3 q})$. Finally, it was found that 1-bromopent-1-yne still reacted with $\mathbf{2 a}$ to afford the desired product $\mathbf{3 r}$ in $75 \%$ isolated yield.

To explore the scope of this method, we next turned our attention to the HIRs, and the result is shown in Table 3. Firstly, a number of $\mathrm{PhI}\left(\mathrm{O}_{2} \mathrm{CR}\right)_{2}$ were readily synthesized through a ligand exchange of DIB with carboxylate acids in boiling toluene. Notably, most of the HIRs derived from aryl carboxylic acid reacted with (bromoethynyl)benzene 1a under optimized conditions to afford the addition products in satisfactory yields $(\mathbf{4 a} \sim \mathbf{4 d})$. The scope was also expanded to HIRs derived from alkyl carboxylic acid, and which produced the addition products in lower yields $(\mathbf{4 e} \sim \mathbf{4 f})$. This result can be explained by an intramolecular competition experiment of BI-OAc with bromoalkyne 1a

Table 2 Scope of bromoalkynes ${ }^{a}$

<smiles>Cc1ccc(C(=O)OC(=CBr)c2ccccc2)cc1</smiles>

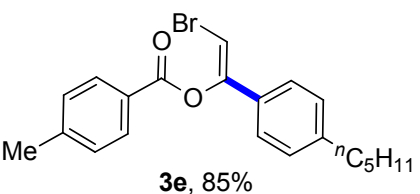
$3 \mathbf{e}, 85 \%$<smiles>COC(=O)OC(=CBr)c1ccc(Br)cc1</smiles>

$3 i, 86 \%$<smiles>Cc1ccc(C(=O)O/C(=C\Br)c2ccc(-c3ccccc3)cc2)cc1</smiles>

$3 \mathrm{~m}, 76 \%$<smiles>Cc1ccc(C(=[OH+])OC(=CBr)c2ccc([N+](=O)[O-])cc2)cc1</smiles><smiles>COc1ccc(C(=CBr)OC(=O)c2ccc(C)cc2)cc1</smiles>
3f, $83 \%$<smiles>Cc1ccc(C(=O)O/C(=C\Br)c2ccc(C#N)cc2)cc1</smiles>

3j, $90 \%$<smiles>Cc1ccc(C(=O)O/C(=C\Br)c2cccc(C)c2)cc1</smiles>

3n, $79 \%$<smiles>Cc1ccc(C(=O)O/C(=C\Br)c2ccsc2)cc1</smiles>

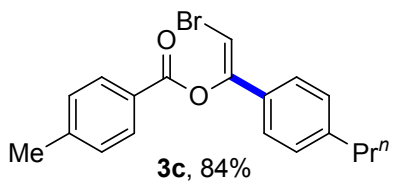<smiles>Cc1ccc(C(=O)OC(=CCBr)c2ccc(Br)cc2)cc1</smiles><smiles>CCOc1ccc(/C(=C/Br)OC(=O)c2ccc(C)cc2)cc1</smiles><smiles>Cc1ccc(C(=O)OC(=CBr)c2ccc(F)cc2)cc1</smiles><smiles>COc1ccc(C(=O)O/C(=C\Br)c2ccc([N+](=O)[O-])cc2)cc1</smiles><smiles>Cc1ccc(C(=O)OC(=CBr)c2cccc(F)c2)cc1</smiles>

3o, $81 \%$<smiles>COc1ccc(C(=O)OC(=CCBr)c2ccc(C(F)(F)F)cc2)cc1</smiles>

3I, $87 \%$<smiles>Cc1ccc(C(=O)O/C(=C\Br)c2cccs2)cc1</smiles>

3p, $68 \%$<smiles>CCCCCCCCCCCCCCCCCCCC(=CCBr)OC(=O)c1ccc(C)cc1</smiles>

${ }^{a}$ Reaction conditions: 1a $(0.2 \mathrm{mmol}), 2(0.2 \mathrm{mmol})$ and $\mathrm{Ag}_{2} \mathrm{CO}_{3}(2.5 \mathrm{~mol} \%)$ in $\mathrm{CH}_{3} \mathrm{CN}(1 \mathrm{~mL})$ at $90{ }^{\circ} \mathrm{C}$ under ambient air for $6 \mathrm{~h} .{ }^{b}$ Isolated yield. 
Table 3 Scope of HIRs and alkynes ${ }^{a}$

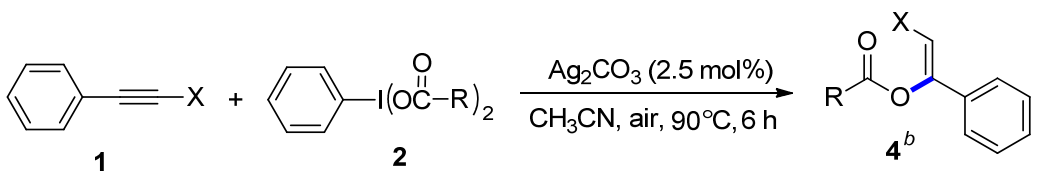<smiles>O=C(OC(=CBr)c1ccccc1)c1ccccc1</smiles>

4a, $74 \%$<smiles>CC(=O)O/C(=C\Br)c1ccccc1</smiles>

4e, $53 \%$<smiles>CC(=O)C=C(OC(C)=O)c1ccccc1</smiles>

$4 \mathbf{i}, 95 \%^{d}$<smiles>CC(C)(C)c1ccc(C(=O)OC(=CBr)c2ccccc2)cc1</smiles>

4b, $81 \%$<smiles>O=C(OC(=CBr)c1ccccc1)c1ccccc1</smiles>

4f, $37 \%$<smiles>CCC(=O)OC(=CC(=O)OC)c1ccccc1</smiles>

$4 \mathrm{j}, 92 \%{ }^{\mathrm{d}}$<smiles>O=C(O/C(=C/Br)c1ccccc1)c1cccc(Cl)c1</smiles>

4c, $77 \%$<smiles>Cc1ccccc1C(=O)O/C(=C\Br)c1ccccc1</smiles>

$4 d, 80 \%$<smiles>CC(=O)O/C(=C\Cl)c1ccccc1</smiles>

4g, $55 \%$<smiles>CC(=O)O/C(=C\I)c1ccccc1</smiles>

4h, $62 \%^{\circ}$<smiles>COC(=O)C=C(OC(=O)c1cccc(Cl)c1)c1ccccc1</smiles><smiles>COC(=O)/C=C(\OC(=O)c1ccc(C(C)(C)C)cc1)c1ccccc1</smiles>

$4 \mathrm{I}, 90 \%^{d}$

${ }^{a}$ Reaction conditions: $1(0.2 \mathrm{mmol}), 2(0.2 \mathrm{mmol})$ and $\mathrm{Ag}_{2} \mathrm{CO}_{3}(2.5 \mathrm{~mol} \%)$ in $\mathrm{CH}_{3} \mathrm{CN}(1 \mathrm{~mL})$ at $90{ }^{\circ} \mathrm{C}$ under ambient air for $6 \mathrm{~h} .{ }^{b}$ Isolated yield. ${ }^{c} 24 \mathrm{~h} .{ }^{d} 10 \mathrm{~h}$.

under standard reaction conditions, generating $\mathbf{5}$ as a major product in $76 \%$ yield (Scheme 2). It was found that replacing the bromide with $\mathrm{Cl}$ or I within alkynes still gave the corresponding products in acceptable yields (4g and $4 \mathrm{~h}$ ).

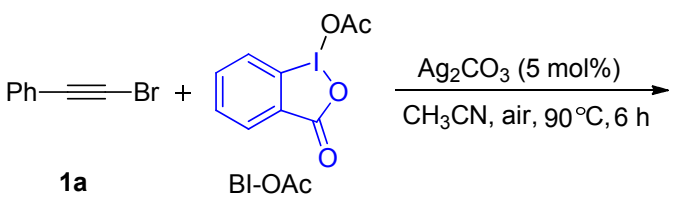<smiles>CC(=O)OC(=C[Br+]Br)c1ccccc1I</smiles>

4e, trace

5, $76 \%$

Scheme 2 Intramolecular competition experiment

Furthermore, methyl 3-phenylpropiolate was employed to react with HIRs under the modified reaction conditions, and which delivered the vinyl esters in excellent yields $(\mathbf{4} \mathbf{i} \sim \mathbf{4 l})$. Then, a gram-scale reaction of $1 \mathbf{a}$ with $2 \mathbf{a}$ was performed under standard conditions, which generated $\mathbf{3 a}$ in $72 \%$ yield (Scheme 3a). Finally, a transformation of $\mathbf{3 i}$ with 1-phenylethyne by Sonogashira reaction was realized, and produced the product 6 in 70\% yield (Scheme $3 \mathrm{~b}$ ).

To reveal the reaction mechanism, several control experiments were subsequently designed and performed under given conditions (Scheme 4). It was found that the reaction of 1-phenylethyne with DIB under air condition did not give the desired product 7 , which demonstrated the critical role of halogens (Scheme 4a). ${ }^{[12]}$ The addition of a radical quencher, 2,2,6,6-tetramethyl-1-piperidinyloxy (TEMPO), into the reaction of $\mathbf{1 a}$ with DIB did not (a) Gram-scale reaction

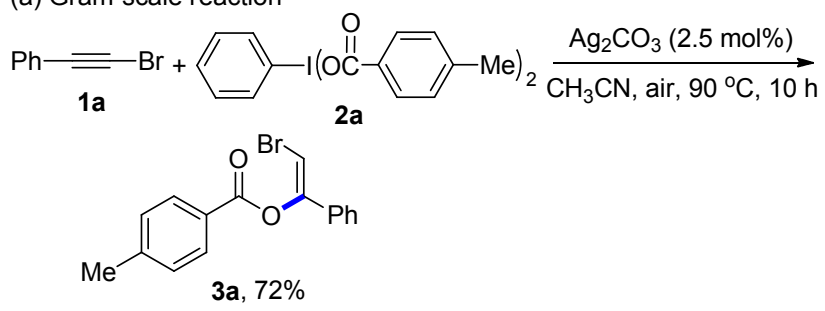

(b) Late-stage transformation

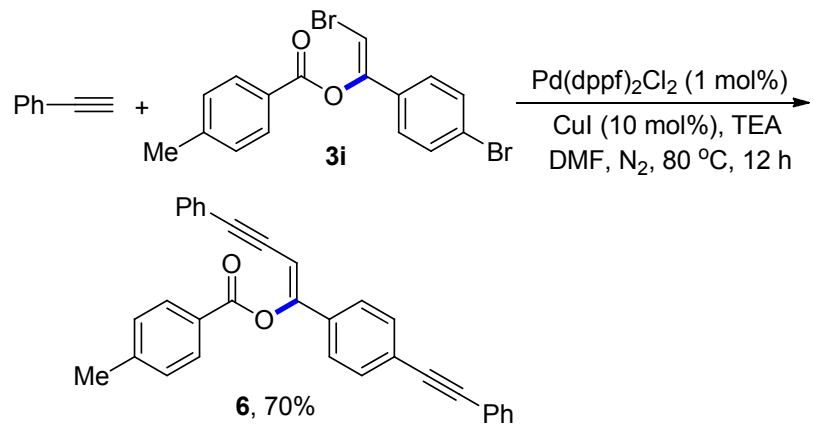

Scheme 3 Gram-scale reaction and late-stage transformation

completely inhibit the process under air atmosphere, generating $4 \mathrm{e}$ in $32 \%$ yield, which indicated a radical pathway was not involved therein (Scheme $4 \mathrm{~b}$ ). The model reaction was carried out in $\mathrm{CH}_{3} \mathrm{CN}$ at $90{ }^{\circ} \mathrm{C}$ under a $\mathrm{N}_{2}$ atmosphere for $6 \mathrm{~h}$, and generated $4 \mathrm{e}$ in $59 \%$ yield, showing the presence of oxygen did not affect the addition event (Scheme 4c). Further, it was found that the selected reaction almost did not proceed in anhydrous $\mathrm{CH}_{3} \mathrm{CN}$ (Scheme $4, \mathrm{~d})$. We then treated this reaction with heavy water, and a $1.86: 1$ ratio of (vinylic) $\mathrm{D} / \mathrm{H}$ was observed by the ${ }^{1} \mathrm{H}$ 
NMR analysis (Scheme 4e). The above result demonstrates that the vinylic hydrogen comes from $\mathrm{H}_{2} \mathrm{O}$ and a protonation step also takes part in this catalytic system.
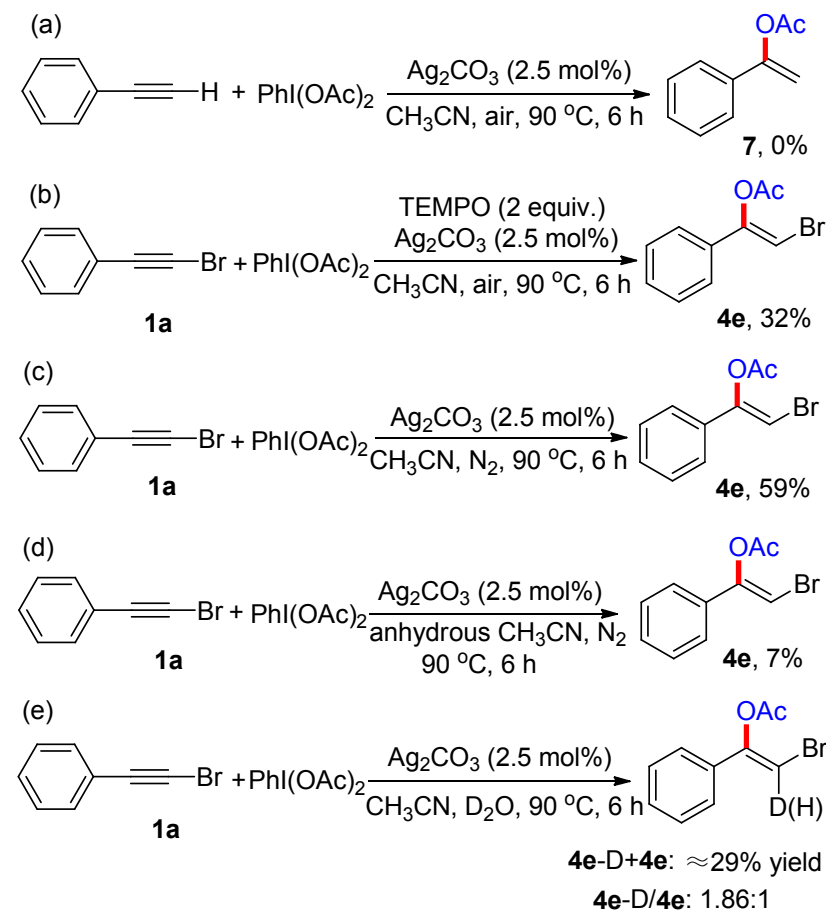

Scheme 4 Mechanistic studies

Based on the above results and previous report, ${ }^{[4-7]}$ a possible reaction mechanism for the silver-catalyzed stereoselective addition reaction of functionalized alkynes with DIB is depicted in Scheme 5. Initially, in the presence of $\mathrm{H}_{2} \mathrm{O}$, DIB interacts with silver(I) via a $\mathrm{I}-\mathrm{O}$ bond cleavage to generate species $\mathbf{A}$ and cationic iodine intermediate B. ${ }^{[12]}$ Then bromoalkyne $\mathbf{1}$ is activated by weak coordination with $\mathbf{A}$ or $\mathbf{B}$ to afford the complex $\mathbf{C}$ or $\mathbf{C}^{\mathbf{c}},{ }^{[4,6 b, 13]}$ followed by a nucleophilic attack of $\mathrm{OAc}^{-}$on the carboncarbon triple bond to give intermediate $\mathbf{D} .^{[13]}$ Finally, the hydrolysis of $\mathbf{D}$ releases a hypervalent iodine compound $\mathbf{E}$ and desired product 3 .

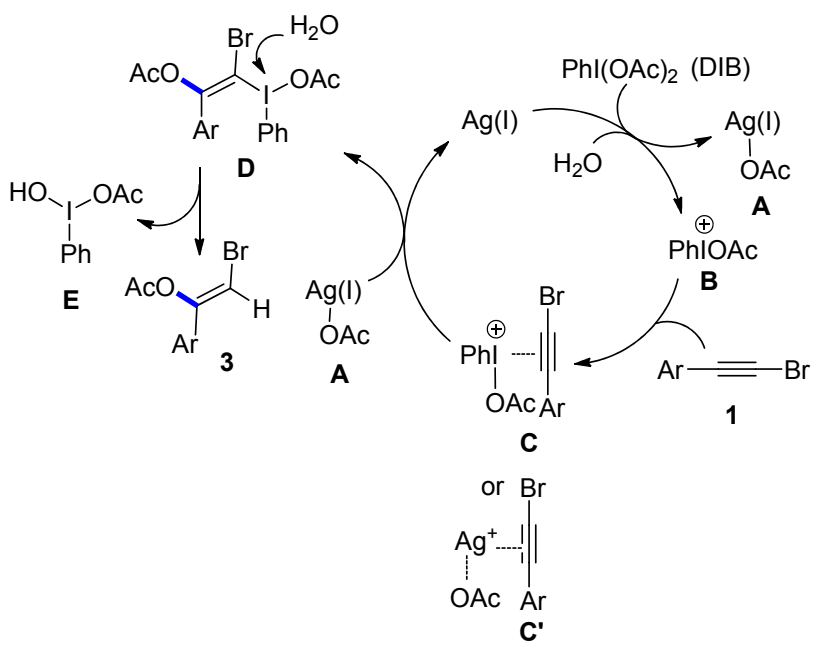

Scheme 5 Possible reaction mechanism

\section{Conclusions}

In summary, a silver-catalyzed stereoselective addition reactions of functionalized alkynes with hypervalent iodine(III) reagents via $\mathrm{I}-\mathrm{O}$ bond cleavage has been developed, which provides an efficient access to vinyl esters in high yields. The control experiment supports that the presence of aryl group considerably affects the cleavage model of $\mathrm{I}-\mathrm{O}$ bond within hypervalent iodine(III) reagents. The further exploration of this strategy using HIRs and transition metal catalysis in synthetic chemistry and mechanistic studies is underway in our laboratory.

\section{Experimental section}

\subsection{General information}

All ${ }^{1} \mathrm{H}$ NMR and ${ }^{13} \mathrm{C}$ NMR spectra were recorded on 400, $600 \mathrm{MHz}$ Bruker FT-NMR spectrometers $(400 \mathrm{MHz}$ and $100 \mathrm{MHz}, 600 \mathrm{MHz}$ and $150 \mathrm{MHz}$, respectively). All chemical shifts are given with reference to tetramethylsilane (TMS) as an internal standard. High resolution mass spectroscopy data of the products were collected on a Thermo Fisher Scientific LTQ FTICR-MS instrument.

The starting materials, such as (bromoethynyl)benzene and hypervalent iodine(III) reagents, are prepared according to the reported methods, respectively. ${ }^{[1-2]}$ All chemical reagents used in this work were purchased from commercial suppliers, Huawei Ruike Chemical company, Energy Chemical company, or Shanghai Chemical Company of China and Aldrich of USA. All solvents were dried and freshly distilled prior to use. Products were purified by flash chromatography on silica gels with petroleum ether/ ethyl acetate $(V: V=10: 1$ to $20: 1)$ as eluent.

4.2 General procedure for the Ag-catalyzed stereoselective addition reaction

A $10 \mathrm{~mL}$ oven-dried reaction tube equipped with a magnetic stirrer bar was charged with alkyne $1(0.20$ $\mathrm{mmol})$, HIRs 2 (0.20 mmol), silver carbonate $(2.5 \mathrm{~mol} \%)$, and freshly distilled $\mathrm{CH}_{3} \mathrm{CN}(1.0 \mathrm{~mL})$ under air atmosphere. The reaction tube was placed in an oil bath and stirred at $90{ }^{\circ} \mathrm{C}$ for $6 \mathrm{~h}$. After the reaction was completed, the mixture was cooled to room temperature, detected by thin-layer chromatography (TLC), and extracted with ethyl acetate $(5 \mathrm{~mL} \times 3)$. The organic layers were combined, dried over anhydrous $\mathrm{MgSO}_{4}$, and concentrated under reduced pressure to yield the crude product, which was further purified by flash chromatography [silica gel, $V$ (petroleum ether) $: V($ ethyl acetate $)=10: 1$ to $20: 1]$, affording the desired product 3 .

(Z)-2-Bromo-1-phenylvinyl 4-methylbenzoate (3a): Yellow liquid, $51.8 \mathrm{mg}$, 82\% yield. ${ }^{1} \mathrm{H}$ NMR (600 MHz, $\left.\mathrm{CDCl}_{3}\right) \delta: 8.13(\mathrm{~d}, J=8.0 \mathrm{~Hz}, 2 \mathrm{H}), 7.49 \sim 7.48(\mathrm{~m}, 2 \mathrm{H})$, $7.36 \sim 7.32(\mathrm{~m}, 5 \mathrm{H}), 6.66(\mathrm{~s}, 1 \mathrm{H}), 2.47(\mathrm{~s}, 3 \mathrm{H}) ;{ }^{13} \mathrm{C} \mathrm{NMR}$ $\left(150 \mathrm{MHz}, \mathrm{CDCl}_{3}\right) \delta: 163.17,150.65,144.81,133.41$, $130.40,129.39,129.34,128.78,125.96,124.92,96.65$, 21.77; HRMS (ESI) calcd for $\mathrm{C}_{16} \mathrm{H}_{13} \mathrm{BrNaO}_{2}[\mathrm{M}+\mathrm{Na}]^{+}$ 338.9991, found 338.9991. 
(Z)-2-Bromo-1-( $p$-tolyl)vinyl 4-methylbenzoate (3b): Pale yellow solid, $52.7 \mathrm{mg}, 80 \%$ yield. m.p. $46.8 \sim$ $47.4{ }^{\circ} \mathrm{C} ;{ }^{1} \mathrm{H}$ NMR (400 MHz, $\left.\mathrm{CDCl}_{3}\right) \delta: 8.13(\mathrm{t}, J=8.4$ $\mathrm{Hz}, 2 \mathrm{H}), 7.36$ (dd, $J=16.0,8.0 \mathrm{~Hz}, 4 \mathrm{H}), 7.16$ (d, $J=8.0$ $\mathrm{Hz}, 2 \mathrm{H}), 6.60$ (s, 1H), 2.47 (s, 3H), 2.34 (s, 3H); ${ }^{13} \mathrm{C} \mathrm{NMR}$ $\left(100 \mathrm{MHz}, \mathrm{CDCl}_{3}\right) \delta: 163.18,150.69,144.72,139.46$, $130.62,130.37,129.45,129.36,126.02,124.82$, 95.61, 21.75, 21.25; HRMS (ESI) calcd for $\mathrm{C}_{17} \mathrm{H}_{14} \mathrm{BrNaO}_{2}[\mathrm{M}+$ $\mathrm{Na}]^{+}$353.0148, found 353.0150.

(Z)-2-Bromo-1-(4-propylphenyl)vinyl 4-methylbenzoate (3c): Pale yellow solid, $60.2 \mathrm{mg}, 84 \%$ yield. m.p. 98.7 $100.4{ }^{\circ} \mathrm{C} ;{ }^{1} \mathrm{H}$ NMR (400 MHz, $\left.\mathrm{CDCl}_{3}\right) \delta: 8.13$ (d, $J=8.0$ $\mathrm{Hz}, 2 \mathrm{H}), 7.38$ (d, $J=8.0 \mathrm{~Hz}, 2 \mathrm{H}), 7.32$ (d, $J=8.0 \mathrm{~Hz}, 2 \mathrm{H})$, $7.15(\mathrm{~d}, J=8.0 \mathrm{~Hz}, 2 \mathrm{H}), 6.61(\mathrm{~s}, 1 \mathrm{H}), 2.59 \sim 2.55(\mathrm{~m}, 2 \mathrm{H})$, $2.47(\mathrm{~s}, 3 \mathrm{H}), 1.65 \sim 1.59(\mathrm{~m}, 2 \mathrm{H}), 0.93(\mathrm{t}, J=7.2 \mathrm{~Hz}, 3 \mathrm{H})$; ${ }^{13} \mathrm{C}$ NMR $\left(100 \mathrm{MHz}, \mathrm{CDCl}_{3}\right) \delta: 163.19,150.71,144.72$, $144.24,130.82,130.39,129.36,128.86,126.04,124.82$, 95.66, 37.75, 24.25, 21.75, 13.73; HRMS (ESI) calcd for $\mathrm{C}_{19} \mathrm{H}_{19} \mathrm{BrNaO}_{2}[\mathrm{M}+\mathrm{Na}]^{+}$381.0461, found 381.0460.

(Z)-2-Bromo-1-(4-butylphenyl)vinyl 4-methylbenzoate (3d): Pale yellow solid, $61.8 \mathrm{mg}, 83 \%$ yield. m.p. $89.2 \sim$ $91.3{ }^{\circ} \mathrm{C} ;{ }^{1} \mathrm{H}$ NMR $\left(400 \mathrm{MHz}, \mathrm{CDCl}_{3}\right) \delta: 8.13$ (d, $J=8.4$ $\mathrm{Hz}, 2 \mathrm{H}), 7.39$ (d, $J=8.0 \mathrm{~Hz}, 2 \mathrm{H}), 7.33$ (d, $J=8.0 \mathrm{~Hz}, 2 \mathrm{H})$, $7.16(\mathrm{~d}, J=8.0 \mathrm{~Hz}, 2 \mathrm{H}), 6.61(\mathrm{~s}, 1 \mathrm{H}), 2.59$ (t, $J=7.6 \mathrm{~Hz}$, 2H), $2.46(\mathrm{~s}, 3 \mathrm{H}), 1.58(\mathrm{t}, J=8.0 \mathrm{~Hz}, 2 \mathrm{H}), 1.34(\mathrm{dd}, J=$ $15.2,7.6 \mathrm{~Hz}, 2 \mathrm{H}), 0.92(\mathrm{t}, J=7.2 \mathrm{~Hz}, 3 \mathrm{H}) ;{ }^{13} \mathrm{C}$ NMR $(100$ $\left.\mathrm{MHz}, \mathrm{CDCl}_{3}\right) \delta: 163.19,150.73,144.72,144.48,130.79$, $130.39,129.36,128.82,126.06,124.84,95.65,35.37$, $33.30,22.27,21.76,13.87$; HRMS (ESI) calcd for $\mathrm{C}_{20} \mathrm{H}_{21^{-}}$ $\mathrm{BrNaO}_{2}[\mathrm{M}+\mathrm{Na}]^{+}$395.0617, found 395.0617.

(Z)-2-Bromo-1-(4-pentylphenyl)vinyl 4-methylbenzoate (3e): Pale yellow solid, $65.6 \mathrm{mg}, 85 \%$ yield. m.p. 101.4 $103.2{ }^{\circ} \mathrm{C} ;{ }^{1} \mathrm{H}$ NMR $\left(600 \mathrm{MHz}, \mathrm{CDCl}_{3}\right) \delta: 8.14(\mathrm{~d}, J=8.0$ $\mathrm{Hz}, 2 \mathrm{H}), 7.39$ (d, $J=8.0 \mathrm{~Hz}, 2 \mathrm{H}), 7.34$ (d, $J=8.0 \mathrm{~Hz}, 2 \mathrm{H})$, $7.16(\mathrm{~d}, J=8.0 \mathrm{~Hz}, 2 \mathrm{H}), 6.61(\mathrm{~s}, 1 \mathrm{H}), 2.59(\mathrm{t}, J=7.6 \mathrm{~Hz}$, $2 \mathrm{H}), 2.47(\mathrm{~s}, 3 \mathrm{H}), 1.61 \sim 1.58(\mathrm{~m}, 2 \mathrm{H}), 1.36 \sim 1.30(\mathrm{~m}$, $4 \mathrm{H}), 0.90(\mathrm{t}, J=6.9 \mathrm{~Hz}, 3 \mathrm{H}) ;{ }^{13} \mathrm{C} \mathrm{NMR}\left(150 \mathrm{MHz}, \mathrm{CDCl}_{3}\right)$ $\delta: 163.25,150.78,144.77,144.56,130.84,130.45,129.42$, $128.87,126.12,124.90,95.71,35.71,31.45,30.90,22.53$, 21.81, 14.02; HRMS (ESI) calcd for $\mathrm{C}_{21} \mathrm{H}_{23} \mathrm{BrNaO}_{2}[\mathrm{M}+$ $\mathrm{Na}]^{+}$409.0774, found 409.0774.

(Z)-2-Bromo-1-(4-methoxyphenyl)vinyl 4-methylbenzoate (3f): Pale yellow solid, $57.5 \mathrm{mg}, 83 \%$ yield. m.p. 101.6 102.6 ${ }^{\circ} \mathrm{C} ;{ }^{1} \mathrm{H}$ NMR $\left(400 \mathrm{MHz}, \mathrm{CDCl}_{3}\right) \delta: 8.13(\mathrm{~d}$, $J=8.0 \mathrm{~Hz}, 2 \mathrm{H}), 7.42$ (d, $J=8.0 \mathrm{~Hz}, 2 \mathrm{H}), 7.33$ (d, $J=7.6$ $\mathrm{Hz}, 2 \mathrm{H}), 6.87$ (d, J=7.6 Hz, 2H), 6.51 (s, 1H), 3.80 (s, $3 \mathrm{H}) ; 2.46(\mathrm{~s}, 3 \mathrm{H}) ;{ }^{13} \mathrm{C} \mathrm{NMR}\left(100 \mathrm{MHz}, \mathrm{CDCl}_{3}\right) \delta: 164.45$, $161.73,151.68,145.97,131.63,130.62,127.68,127.34$, 115.44, 95.72, 56.56, 23.00; HRMS (ESI) calcd for $\mathrm{C}_{17} \mathrm{H}_{16} \mathrm{BrNaO}_{3}[\mathrm{M}+\mathrm{Na}]^{+}$369.0097, found 369.0097.

(Z)-2-Bromo-1-(4-ethoxyphenyl)vinyl 4-methylbenzoate (3g): Pale yellow solid, $61.9 \mathrm{mg}, 86 \%$ yield. m.p. 102.7 $103.2{ }^{\circ} \mathrm{C} ;{ }^{1} \mathrm{H}$ NMR (400 MHz, $\left.\mathrm{CDCl}_{3}\right) \delta: 8.13(\mathrm{~d}, J=8.0$ $\mathrm{Hz}, 2 \mathrm{H}), 7.40$ (d, $J=8.8 \mathrm{~Hz}, 2 \mathrm{H}), 7.33$ (d, $J=8.0 \mathrm{~Hz}, 2 \mathrm{H})$, $6.85(\mathrm{~d}, J=8.8 \mathrm{~Hz}, 2 \mathrm{H}), 6.51(\mathrm{~s}, 1 \mathrm{H}), 4.02(\mathrm{dd}, J=13.0$, $8.4 \mathrm{~Hz}, 2 \mathrm{H}), 2.46(\mathrm{~s}, 3 \mathrm{H}), 1.40(\mathrm{t}, J=6.8 \mathrm{~Hz}, 3 \mathrm{H}) ;{ }^{13} \mathrm{C}$ NMR $\left(100 \mathrm{MHz}, \mathrm{CDCl}_{3}\right) \delta: 163.19,159.85,150.45$,
$144.70,130.38,129.36,126.39,126.08,125.86,114.67$, 94.35, 63.53, 21.76, 14.70; HRMS (ESI) calcd for $\mathrm{C}_{18} \mathrm{H}_{17^{-}}$ $\mathrm{BrNaO}_{3}[\mathrm{M}+\mathrm{Na}]^{+}$383.0253, found 383.0253.

(Z)-2-Bromo-1-(4-fluorophenyl)vinyl 4-methylbenzoate (3h): Pale yellow solid, $59.5 \mathrm{mg}, 89 \%$ yield. m.p. 76.2 $77.5{ }^{\circ} \mathrm{C} ;{ }^{1} \mathrm{H}$ NMR $\left(600 \mathrm{MHz}, \mathrm{CDCl}_{3}\right) \delta: 8.12$ (d, $J=8.0$ $\mathrm{Hz}, 2 \mathrm{H}), 7.48 \sim 7.45(\mathrm{~m}, 2 \mathrm{H}), 7.33(\mathrm{~d}, J=7.8 \mathrm{~Hz}, 2 \mathrm{H})$, $7.04(\mathrm{t}, J=9.0 \mathrm{~Hz}, 2 \mathrm{H}), 6.59(\mathrm{~s}, 1 \mathrm{H}), 2.46(\mathrm{~s}, 3 \mathrm{H}) ;{ }^{13} \mathrm{C}$ NMR $\left(150 \mathrm{MHz}, \mathrm{CDCl}_{3}\right) \delta: 163.25(\mathrm{~d}, J=249.9 \mathrm{~Hz})$, 162.42, 149.81, 144.95, 130.40, 129.75 (d, $J=3.2 \mathrm{~Hz})$, $129.43,126.94$ (d, $J=8.1 \mathrm{~Hz}), 125.80,115.89$ (d, $J=21.8$ $\mathrm{Hz}), 96.38,21.77 ;{ }^{19} \mathrm{~F} \mathrm{NMR}\left(600 \mathrm{MHz}, \mathrm{CDCl}_{3}\right) \quad \delta$ : -111.2 ; HRMS (ESI) calcd for $\mathrm{C}_{16} \mathrm{H}_{12} \mathrm{BrFNaO}_{2}[\mathrm{M}+$ $\mathrm{Na}]^{+}$356.9897, found 356.9899.

(Z)-2-Bromo-1-(4-bromophenyl)vinyl 4-methylbenzoate (3i): Pale yellow solid, $67.7 \mathrm{mg}, 86 \%$ yield. m.p. 83.3 $84.8{ }^{\circ} \mathrm{C}$; ${ }^{1} \mathrm{H}$ NMR $\left(400 \mathrm{MHz}, \mathrm{CDCl}_{3}\right) \delta: 8.11$ (d, $J=8.0$ $\mathrm{Hz}, 2 \mathrm{H}), 7.48$ (d, $J=8.8 \mathrm{~Hz}, 2 \mathrm{H}), 7.34$ (dd, $J=8.5,2.5 \mathrm{~Hz}$, $4 \mathrm{H}), 6.67(\mathrm{~s}, 1 \mathrm{H}), 2.47(\mathrm{~s}, 3 \mathrm{H}) ;{ }^{13} \mathrm{C} \mathrm{NMR}(100 \mathrm{MHz}$, $\left.\mathrm{CDCl}_{3}\right) \delta: 163.08,149.81,144.99,132.45,131.98,130.40$, 129.44, 126.44, 125.73, 123.53, 97.33, 21.77; HRMS (ESI) calcd for $\mathrm{C}_{16} \mathrm{H}_{12} \mathrm{Br}_{2} \mathrm{NaO}_{2}[\mathrm{M}+\mathrm{Na}]^{+}$416.9096, found 416.9097.

(Z)-2-Bromo-1-(4-cyanophenyl)vinyl 4-methylbenzoate (3j): Pale yellow solid, $61.4 \mathrm{mg}, 90 \%$ yield. m.p. 156.3 $157.7{ }^{\circ} \mathrm{C} ;{ }^{1} \mathrm{H}$ NMR $\left(400 \mathrm{MHz}, \mathrm{CDCl}_{3}\right) \delta: 8.11$ (d, $J=8.0$ $\mathrm{Hz}, 2 \mathrm{H}), 7.64$ (d, $J=8.4 \mathrm{~Hz}, 2 \mathrm{H}), 7.57$ (d, $J=8.4 \mathrm{~Hz}, 2 \mathrm{H})$, 7.34 (d, $J=8.0 \mathrm{~Hz}, 2 \mathrm{H}), 6.84(\mathrm{~s}, 1 \mathrm{H}), 2.47(\mathrm{~s}, 3 \mathrm{H}) ;{ }^{13} \mathrm{C}$ NMR $\left(100 \mathrm{MHz}, \mathrm{CDCl}_{3}\right) \delta: 163.02,149.14,145.30$, $137.62,132.62,130.44,129.53,125.38,118.24,112.80$, 100.42, 21.78; HRMS (ESI) calcd for $\mathrm{C}_{17} \mathrm{H}_{12} \mathrm{BrNNaO}_{2}$ $[\mathrm{M}+\mathrm{Na}]^{+}$363.9944, found 363.9942 .

(Z)-2-Bromo-1-(4-nitrophenyl)vinyl 4-methylbenzoate (3k): Pale yellow solid, $66.4 \mathrm{mg}, 92 \%$ yield. m.p. 148.0 $149.9{ }^{\circ} \mathrm{C} ;{ }^{1} \mathrm{H}$ NMR (400 MHz, $\mathrm{CDCl}_{3}$ ) $\delta: 8.21$ (d, $J=8.8$ $\mathrm{Hz}, 2 \mathrm{H}), 8.12$ (d, $J=8.4 \mathrm{~Hz}, 2 \mathrm{H}), 7.64$ (d, $J=9.2 \mathrm{~Hz}, 2 \mathrm{H})$, $7.35(\mathrm{~d}, J=8.0 \mathrm{~Hz}, 2 \mathrm{H}), 6.91(\mathrm{~s}, 1 \mathrm{H}), 2.48(\mathrm{~s}, 3 \mathrm{H}) ;{ }^{13} \mathrm{C}$ NMR $\left(100 \mathrm{MHz}, \mathrm{CDCl}_{3}\right) \delta: 163.00,148.91,147.92$, $145.35,139.34,130.43,129.54,125.58,125.30,124.12$, 101.10, 21.78; HRMS (ESI) calcd for $\mathrm{C}_{16} \mathrm{H}_{12} \mathrm{BrNNaO}_{4}$ $[\mathrm{M}+\mathrm{Na}]^{+}$383.9842, found 383.9842 .

(Z)-2-Bromo-1-(4-(trifluoromethyl)phenyl)vinyl 4methylbenzoate (31): Pale yellow solid, $66.8 \mathrm{mg}, 87 \%$ yield. m.p. $75.3 \sim 77.2{ }^{\circ} \mathrm{C} ;{ }^{1} \mathrm{H}$ NMR $\left(400 \mathrm{MHz}, \mathrm{CDCl}_{3}\right) \delta$ : $8.12(\mathrm{~d}, J=8.0 \mathrm{~Hz}, 2 \mathrm{H}), 7.63 \sim 7.57(\mathrm{~m}, 4 \mathrm{H}), 7.35(\mathrm{~d}, J=$ $8.0 \mathrm{~Hz}, 2 \mathrm{H}), 6.79(\mathrm{~s}, 1 \mathrm{H}), 2.47(\mathrm{~s}, 3 \mathrm{H}) ;{ }^{13} \mathrm{C}$ NMR $(150$ $\left.\mathrm{MHz}, \mathrm{CDCl}_{3}\right) \delta: 163.11,149.53,145.17,136.83,131.12$ (q, $J=32.4 \mathrm{~Hz}), 130.78,130.44,129.50,123.79$ (q, $J=$ $270.4 \mathrm{~Hz}$ ), 125.85 (q, $J=3.0 \mathrm{~Hz}$ ), 125.188, 99.15, 21.79; ${ }^{19} \mathrm{~F}$ NMR $\left(600 \mathrm{MHz}, \mathrm{CDCl}_{3}\right) \delta$ : -62.8 ; HRMS (ESI) calcd for $\mathrm{C}_{17} \mathrm{H}_{13} \mathrm{BrF}_{3} \mathrm{NaO}_{2}[\mathrm{M}+\mathrm{Na}]^{+}$406.9865, found 406.9865 .

(Z)-1-([1,1'-Biphenyl]-4-yl)-2-bromovinyl 4-methylbenzoate $(\mathbf{3 m})$ : Pale yellow solid, $59.9 \mathrm{mg}, 76 \%$ yield. m.p. $123.3 \sim 124.6{ }^{\circ} \mathrm{C} ;{ }^{1} \mathrm{H}$ NMR (400 MHz, $\left.\mathrm{CDCl}_{3}\right) \delta$ : $8.16(\mathrm{~d}, J=8.4 \mathrm{~Hz}, 2 \mathrm{H}), 7.60 \sim 7.54(\mathrm{~m}, 6 \mathrm{H}), 7.44(\mathrm{t}, J=$ $7.2 \mathrm{~Hz}, 2 \mathrm{H}), 7.36(\mathrm{t}, J=8.8 \mathrm{~Hz}, 3 \mathrm{H}), 6.72(\mathrm{~s}, 1 \mathrm{H}), 2.48$ (s, 
$3 \mathrm{H}) ;{ }^{13} \mathrm{C} \mathrm{NMR}\left(100 \mathrm{MHz}, \mathrm{CDCl}_{3}\right) \delta: 163.24,150.42$, $144.86,142.16,140.18,132.26,130.44,129.43,128.84$, $127.68,127.49,127.00,125.96,125.31,96.62,21.78$; HRMS (ESI) calcd for $\mathrm{C}_{22} \mathrm{H}_{17} \mathrm{BrNaO}_{2} \quad\left[\mathrm{M}+\mathrm{Na}{ }^{+}\right.$ 415.0304, found 415.0306.

(Z)-2-Bromo-1-( $m$-tolyl)vinyl 4-methylbenzoate (3n): Pale yellow solid, $52.1 \mathrm{mg}, 79 \%$ yield. m.p. 45.8 $47.9{ }^{\circ} \mathrm{C} ;{ }^{1} \mathrm{H}$ NMR $\left(400 \mathrm{MHz}, \mathrm{CDCl}_{3}\right) \delta: 8.18(\mathrm{~d}, J=8.4$ $\mathrm{Hz}, 2 \mathrm{H}), 7.38$ (d, $J=8.0 \mathrm{~Hz}, 2 \mathrm{H}), 7.33$ (d, $J=6.4 \mathrm{~Hz}, 2 \mathrm{H})$, 7.29 (d, $J=7.6 \mathrm{~Hz}, 1 \mathrm{H}), 7.21$ (d, $J=7.2 \mathrm{~Hz}, 1 \mathrm{H}), 6.68$ (s, 1H), 2.51 (s, 3H), $2.39(\mathrm{~s}, 3 \mathrm{H}) ;{ }^{13} \mathrm{C} \mathrm{NMR}(100 \mathrm{MHz}$, $\mathrm{CDCl}_{3}$ ) $\delta: 163.19,150.79,144.75,138.47,133.36,130.39$, $130.18,129.37,128.66,126.01,125.53,122.09$, 96.38, 21.74, 21.39; HRMS (ESI) calcd for $\mathrm{C}_{17} \mathrm{H}_{15} \mathrm{BrNaO}_{2}[\mathrm{M}+$ $\mathrm{Na}]^{+}$353.0148, found 353.0148.

(Z)-2-Bromo-1-(3-fluorophenyl)vinyl 4-methylbenzoate (3o): pale yellow solid, $54.1 \mathrm{mg}, 81 \%$ yield. m.p. 78.0 $79.2{ }^{\circ} \mathrm{C} ;{ }^{1} \mathrm{H}$ NMR $\left(400 \mathrm{MHz}, \mathrm{CDCl}_{3}\right) \delta: 8.13(\mathrm{~d}, J=8.0$ $\mathrm{Hz}, 2 \mathrm{H}), 7.42(\mathrm{td}, J=7.7,1.6 \mathrm{~Hz}, 1 \mathrm{H}), 7.36 \sim 7.29(\mathrm{~m}$, $3 \mathrm{H}), 7.14 \sim 7.09(\mathrm{~m}, 2 \mathrm{H}), 6.91(\mathrm{~s}, 1 \mathrm{H}), 2.46(\mathrm{~s}, 3 \mathrm{H}) ;{ }^{13} \mathrm{C}$ NMR (100 MHz, $\left.\mathrm{CDCl}_{3}\right) \delta: 163.08,159.48$ (d, $J=250.3$ $\mathrm{Hz}), 145.07$ (d, $J=4.3 \mathrm{~Hz}), 144.86,130.58$ (d, $J=8.7 \mathrm{~Hz})$, 130.40, 129.39, 127.72 (d, $J=1.7 \mathrm{~Hz}), 125.80,124.36$ (d, $J=3.5 \mathrm{~Hz}), 121.26(\mathrm{~d}, J=11.4 \mathrm{~Hz}), 116.39$ (d, $J=22.5$ $\mathrm{Hz}), 101.81$ (d, $J=13.7 \mathrm{~Hz}), 21.75 ;{ }^{19} \mathrm{~F}$ NMR $(600 \mathrm{MHz}$, $\left.\mathrm{CDCl}_{3}\right) \delta$ : -111.9 ; HRMS (ESI) calcd for $\mathrm{C}_{16} \mathrm{H}_{12} \mathrm{Br}-$ $\mathrm{FNaO}_{2}[\mathrm{M}+\mathrm{Na}]^{+}$356.9897, found 356.9897.

(Z)-2-Bromo-1-(thiophen-2-yl)vinyl 4-methylbenzoate (3p): Pale yellow solid, $43.8 \mathrm{mg}$, 68\% yield. m.p. 86.1 $87.9{ }^{\circ} \mathrm{C} ;{ }^{1} \mathrm{H}$ NMR $\left(400 \mathrm{MHz}, \mathrm{CDCl}_{3}\right) \delta: 8.12(\mathrm{~d}, J=8.4$ $\mathrm{Hz}, 2 \mathrm{H}), 7.33$ (d, $J=8.0 \mathrm{~Hz}, 2 \mathrm{H}), 7.28$ (dd, $J=4.8,0.8 \mathrm{~Hz}$, $1 \mathrm{H}), 7.15(\mathrm{dd}, J=4.0,0.8 \mathrm{~Hz}, 1 \mathrm{H}), 6.98(\mathrm{dd}, J=5.2,4.0$ $\mathrm{Hz}, 1 \mathrm{H}), 6.59$ (s, 1H), 2.46 (s, 3H); ${ }^{13} \mathrm{C}$ NMR $(100 \mathrm{MHz}$, $\left.\mathrm{CDCl}_{3}\right) \delta: 162.87,145.65,144.91,136.56,130.44,129.39$, 127.57, 126.36, 125.74, 125.15, 95.51, 21.77; HRMS (ESI) calcd for $\left.\mathrm{C}_{14} \mathrm{H}_{11} \mathrm{Br} \mathrm{NaO} \mathrm{Na}_{2} \mathrm{~S}+\mathrm{Na}\right]^{+}$344.9555, found 344.9556.

(Z)-2-Bromo-1-(thiophen-3-yl)vinyl 4-methylbenzoate (3q): Pale yellow solid, $39.3 \mathrm{mg}, 61 \%$ yield. m.p. 88.6 $89.3{ }^{\circ} \mathrm{C} ;{ }^{1} \mathrm{H}$ NMR $\left(400 \mathrm{MHz}, \mathrm{CDCl}_{3}\right) \delta: 8.12(\mathrm{~d}, J=8.4$ $\mathrm{Hz}, 2 \mathrm{H}), 7.34 \sim 7.31(\mathrm{~m}, 4 \mathrm{H}), 7.19(\mathrm{dd}, J=4.8,1.2 \mathrm{~Hz}$, $1 \mathrm{H}), 6.61(\mathrm{~s}, 1 \mathrm{H}), 2.47(\mathrm{~s}, 3 \mathrm{H}) ;{ }^{13} \mathrm{C}$ NMR $(100 \mathrm{MHz}$, $\left.\mathrm{CDCl}_{3}\right) \delta: 163.03,146.88,144.85,135.01,130.39,129.39$, 126.93, 125.87, 124.20, 122.36, 96.27, 21.76; HRMS (ESI) calcd for $\mathrm{C}_{14} \mathrm{H}_{11} \mathrm{BrNaO}_{2} \mathrm{~S}[\mathrm{M}+\mathrm{Na}]^{+}$344.9555, found 344.9555.

(Z)-1-Bromohept-1-en-2-yl 4-methylbenzoate (3r): Pale yellow liquid, $46.5 \mathrm{mg}, 75 \%$ yield. ${ }^{1} \mathrm{H}$ NMR $(600 \mathrm{MHz}$, $\left.\mathrm{CDCl}_{3}\right) \delta: 8.03(\mathrm{~d}, J=8.4 \mathrm{~Hz}, 2 \mathrm{H}), 7.29(\mathrm{~d}, J=7.8 \mathrm{~Hz}$, $2 \mathrm{H}), 5.91(\mathrm{~s}, 1 \mathrm{H}), 2.44(\mathrm{~s}, 3 \mathrm{H}), 2.42 \sim 2.39(\mathrm{~m}, 2 \mathrm{H}), 1.53 \sim$ $1.51(\mathrm{~m}, 2 \mathrm{H}), 1.33 \sim 1.31(\mathrm{~m}, 4 \mathrm{H}), 0.90 \sim 0.87(\mathrm{~m}, 3 \mathrm{H})$; ${ }^{13} \mathrm{C}$ NMR $\left(150 \mathrm{MHz}, \mathrm{CDCl}_{3}\right) \delta: 163.19,153.27,144.48$, $130.20,129.26,126.31,93.70,33.66,31.09,25.85,22.30$, 21.73, 13.91; HRMS (ESI) calcd for $\mathrm{C}_{15} \mathrm{H}_{19} \mathrm{BrNaO}_{2}[\mathrm{M}+$ $\mathrm{Na}]^{+}$333.0461, found 333.0463.

For the synthesis of compound 4 , see the general procedure for compound $\mathbf{3}$.
(Z)-2-Bromo-1-phenylvinyl benzoate (4a): ${ }^{[14]}$ Pale yellow solid, $44.7 \mathrm{mg}, 74 \%$ yield. m.p. $45.8 \sim 47.3{ }^{\circ} \mathrm{C} ;{ }^{1} \mathrm{H}$ NMR $\left(400 \mathrm{MHz} \mathrm{CDCl}_{3}\right) \delta: 8.25(\mathrm{dd}, J=8.0,0.8 \mathrm{~Hz}, 2 \mathrm{H})$, $7.67(\mathrm{t}, J=7.6 \mathrm{~Hz}, 1 \mathrm{H}), 7.54(\mathrm{t}, J=7.8 \mathrm{~Hz}, 2 \mathrm{H}), 7.51 \sim$ $7.48(\mathrm{~m}, 2 \mathrm{H}), 7.38 \sim 7.36(\mathrm{~m}, 3 \mathrm{H}), 6.68(\mathrm{~s}, 1 \mathrm{H}) ;{ }^{13} \mathrm{C} \mathrm{NMR}$ $\left(100 \mathrm{MHz}, \mathrm{CDCl}_{3}\right) \delta: 163.10,150.63,133.86,133.31$, $130.33,129.39,128.79,128.74,128.67,124.92,96.71$.

(Z)-2-Bromo-1-phenylvinyl 4-(tert-butyl)benzoate (4b): White solid, $58.0 \mathrm{mg}, 81 \%$ yield. m.p. $82.4 \sim 83.7{ }^{\circ} \mathrm{C} ;{ }^{1} \mathrm{H}$ NMR (400 MHz, $\left.\mathrm{CDCl}_{3}\right) \delta: 8.17(\mathrm{~d}, J=8.8 \mathrm{~Hz}, 2 \mathrm{H}), 7.55$ $(\mathrm{d}, J=8.4 \mathrm{~Hz}, 2 \mathrm{H}), 7.50 \sim 7.47(\mathrm{~m}, 2 \mathrm{H}), 7.36 \sim 7.33(\mathrm{~m}$, $3 \mathrm{H}), 6.66(\mathrm{~s}, 1 \mathrm{H}), 1.38(\mathrm{~s}, 9 \mathrm{H}) ;{ }^{13} \mathrm{C} \mathrm{NMR}(100 \mathrm{MHz}$, $\left.\mathrm{CDCl}_{3}\right) \delta: 163.09,157.76,150.68,133.46,130.29,129.35$, $128.78,125.95,125.70,124.95,96.64,35.24,31.09$; HRMS (ESI) calcd for $\mathrm{C}_{19} \mathrm{H}_{19} \mathrm{BrNaO}_{2}[\mathrm{M}+\mathrm{Na}]$ 381.0461, found 381.0461.

(Z)-2-Bromo-1-phenylvinyl 3-chlorobenzoate (4c): Pale yellow solid, $51.7 \mathrm{mg}, 77 \%$ yield. m.p. $79.9 \sim 80.9{ }^{\circ} \mathrm{C} ;{ }^{1} \mathrm{H}$ NMR (400 MHz, $\left.\mathrm{CDCl}_{3}\right) \delta: 8.23(\mathrm{t}, J=1.6 \mathrm{~Hz}, 1 \mathrm{H}), 8.13$ $(\mathrm{dt}, J=8.0 \mathrm{~Hz}, 1.2 \mathrm{~Hz}, 1 \mathrm{H}), 7.66 \sim 7.63(\mathrm{~m}, 1 \mathrm{H}), 7.50 \sim$ 7.46 (m, 3H), 7.37 (dd, $J=6.0,2.4 \mathrm{~Hz}, 3 \mathrm{H}), 6.69$ (s, 1H); ${ }^{13} \mathrm{C}$ NMR $\left(100 \mathrm{MHz}, \mathrm{CDCl}_{3}\right) \delta: 161.91,150.47,134.87$, $133.90,133.01,130.44,130.26,130.01,129.51,128.83$, 128.42, 124.89, 96.89; HRMS (ESI) calcd for $\mathrm{C}_{15} \mathrm{H}_{10} \mathrm{Br}-$ $\mathrm{ClNaO}_{2}[\mathrm{M}+\mathrm{Na}]^{+}$358.9445, found 358.9445.

(Z)-2-Bromo-1-phenylvinyl 2-methylbenzoate (4d): Pale yellow solid, $50.5 \mathrm{mg}, 80 \%$ yield. m.p. $47.8 \sim 48.3{ }^{\circ} \mathrm{C} ;{ }^{1} \mathrm{H}$ NMR (400 MHz, $\left.\mathrm{CDCl}_{3}\right) \delta: 8.29(\mathrm{~d}, J=8.0 \mathrm{~Hz}, 1 \mathrm{H})$, $7.54 \sim 7.50(\mathrm{~m}, 3 \mathrm{H}), 7.38 \sim 7.33(\mathrm{~m}, 5 \mathrm{H}), 6.67(\mathrm{~s}, 1 \mathrm{H})$, $2.68(\mathrm{~s}, 3 \mathrm{H}) ;{ }^{13} \mathrm{C}$ NMR $\left(100 \mathrm{MHz}, \mathrm{CDCl}_{3}\right) \delta: 163.50$, $150.68,141.66,133.51,132.97,131.98,131.33,129.34$, 128.80, 127.75, 125.96, 124.92, 96.63, 21.86; HRMS (ESI) calcd for $\mathrm{C}_{16} \mathrm{H}_{13} \mathrm{BrNaO}_{2}[\mathrm{M}+\mathrm{Na}]^{+}$338.9991, found 338.9990 .

(Z)-2-Bromo-1-phenylvinyl acetate $(4 \mathbf{e})^{[4 a]}$ : Yellow liquid, $25.4 \mathrm{mg}, 53 \%$ yield. ${ }^{1} \mathrm{H}$ NMR (400 $\left.\mathrm{MHz}, \mathrm{CDCl}_{3}\right) \delta$ : $7.44 \sim 7.40(\mathrm{~m}, 2 \mathrm{H}), 7.38 \sim 7.35(\mathrm{~m}, 3 \mathrm{H}), 6.55(\mathrm{~s}, 1 \mathrm{H})$, $2.34(\mathrm{~s}, 3 \mathrm{H}) ;{ }^{13} \mathrm{C} \mathrm{NMR}\left(100 \mathrm{MHz}, \mathrm{CDCl}_{3}\right) \delta$ : 167.23, 150.50, 133.29, 129.39, 128.77, 124.91, 96.55, 20.57.

(Z)-2-Bromo-1-( $p$-tolyl)vinyl heptanoate (4f): Yellow liquid, $23.9 \mathrm{mg}, 37 \%$ yield. ${ }^{1} \mathrm{H}$ NMR $\left(400 \mathrm{MHz}, \mathrm{CDCl}_{3}\right) \delta$ : 7.32 (d, $J=8.8 \mathrm{~Hz}, 2 \mathrm{H}), 6.85$ (d, $J=8.8 \mathrm{~Hz}, 2 \mathrm{H}), 6.39$ (s, $1 \mathrm{H}), 4.03$ (q, $J=7.2 \mathrm{~Hz}, 2 \mathrm{H}), 2.59$ (t, $J=7.6 \mathrm{~Hz}, 2 \mathrm{H}), 1.78$ $(\mathrm{q}, J=7.2 \mathrm{~Hz}, 2 \mathrm{H}), 1.44 \sim 1.34(\mathrm{~m}, 7 \mathrm{H}), 0.93(\mathrm{t}, J=7.2$ $\mathrm{Hz}, 3 \mathrm{H}) ;{ }^{13} \mathrm{C} \mathrm{NMR}\left(100 \mathrm{MHz}, \mathrm{CDCl}_{3}\right) \delta: 170.11,159.83$, $150.28,126.37,125.89,114.62,94.17,63.53,33.99,31.25$, 24.52, 22.27, 14.70, 13.87; HRMS (ESI) calcd for $\mathrm{C}_{16} \mathrm{H}_{21} \mathrm{BrNaO}_{2}[\mathrm{M}+\mathrm{Na}]^{+}$347.0617, found 347.0616.

(Z)-2-Chloro-1-phenylvinyl acetate (4g): ${ }^{[4 a]}$ Pale yellow liquid, $21.5 \mathrm{mg}, 55 \%$ yield. ${ }^{1} \mathrm{H} \mathrm{NMR}\left(600 \mathrm{MHz}, \mathrm{CDCl}_{3}\right) \delta$ : $7.39 \sim 7.36(\mathrm{~m}, 5 \mathrm{H}), 6.45(\mathrm{~s}, 1 \mathrm{H}), 2.34(\mathrm{~s}, 3 \mathrm{H}) ;{ }^{13} \mathrm{C} \mathrm{NMR}$ $\left(150 \mathrm{MHz}, \mathrm{CDCl}_{3}\right) \delta: 167.33,148.41,132.76,129.35$, 128.76, 124.70, 107.98, 20.46.

(Z)-2-Iodo-1-phenylvinyl acetate $(4 \mathbf{h}):^{[4 a]}$ Pale yellow liquid, $35.7 \mathrm{mg}, 62 \%$ yield. ${ }^{1} \mathrm{H}$ NMR $\left(600 \mathrm{MHz}, \mathrm{CDCl}_{3}\right) \delta$ : 7.42 (s, 2H), 7.35 (s, 3H), $6.63(\mathrm{~s}, 1 \mathrm{H}), 2.35(\mathrm{~s}, 3 \mathrm{H}) ;{ }^{13} \mathrm{C}$ NMR (150 MHz, $\left.\mathrm{CDCl}_{3}\right) \delta: 167.33,155.04,133.66$, 
129.39, 128.73, 125.15, 68.28, 20.98.

(Z)-Methyl-3-acetoxy-3-phenylacrylate (4i): Pale yellow liquid, $41.8 \mathrm{mg}, 95 \%$ yield. ${ }^{1} \mathrm{H}$ NMR $\left(600 \mathrm{MHz}, \mathrm{CDCl}_{3}\right) \delta$ : 7.59 (d, $J=7.8 \mathrm{~Hz}, 2 \mathrm{H}), 7.44(\mathrm{~d}, J=6.6 \mathrm{~Hz}, 1 \mathrm{H}), 7.41$ (t, $J=7.2 \mathrm{~Hz}, 2 \mathrm{H}), 6.28$ (s, 1H), 3.74 (s, 3H), 2.40 (s, 3H); ${ }^{13} \mathrm{C}$ NMR $\left(150 \mathrm{MHz}, \mathrm{CDCl}_{3}\right) \delta: 168.12,164.60,158.31$, 133.20, 131.06, 128.80, 125.90, 105.57, 51.49, 20.92; HRMS (ESI) calcd for $\mathrm{C}_{12} \mathrm{H}_{13} \mathrm{O}_{4}[\mathrm{M}+\mathrm{H}]^{+}$221.0808, found 221.0808 .

(Z)-3-Methoxy-3-oxo-1-phenylprop-1-en-1-yl butyrate (4j): Pale yellow liquid, $45.6 \mathrm{mg}, 92 \%$ yield. ${ }^{1} \mathrm{H} \mathrm{NMR}$ $\left(600 \mathrm{MHz}, \mathrm{CDCl}_{3}\right) \delta: 7.58(\mathrm{~d}, J=7.2 \mathrm{~Hz}, 2 \mathrm{H}), 7.45 \sim 7.39$ $(\mathrm{m}, 3 \mathrm{H}), 6.28(\mathrm{~s}, 1 \mathrm{H}), 3.74(\mathrm{~s}, 3 \mathrm{H}), 2.68(\mathrm{t}, J=7.8 \mathrm{~Hz}, 2 \mathrm{H})$, $1.86 \sim 1.80(\mathrm{~m}, 2 \mathrm{H}), 1.06(\mathrm{t}, J=7.2 \mathrm{~Hz}, 3 \mathrm{H}) ;{ }^{13} \mathrm{C} \mathrm{NMR}$ $\left(150 \mathrm{MHz}, \mathrm{CDCl}_{3}\right) \delta: 170.64,164.56,158.29,133.46$, 130.95, 128.77, 125.89, 105.73, 51.43, 36.01, 18.04, 13.70; HRMS (ESI) calcd for $\mathrm{C}_{14} \mathrm{H}_{17} \mathrm{O}_{4}[\mathrm{M}+\mathrm{H}]^{+}$249.1121, found 249.1120 .

(Z)-3-Methoxy-3-oxo-1-phenylprop-1-en-1-yl 3-chlorobenzoate (4k): White solid, $60.7 \mathrm{mg}, 96 \%$ yield. m.p. $146.1 \sim 147.4{ }^{\circ} \mathrm{C} ;{ }^{1} \mathrm{H}$ NMR $\left(600 \mathrm{MHz}, \mathrm{CDCl}_{3}\right) \delta: 7.74(\mathrm{~s}$, $1 \mathrm{H}), 7.73(\mathrm{~d}, J=7.8 \mathrm{~Hz}, 1 \mathrm{H}), 7.25 \sim 7.22(\mathrm{~m}, 2 \mathrm{H}), 7.10 \sim$ $7.00(\mathrm{~m}, 5 \mathrm{H}), 6.02(\mathrm{~s}, 1 \mathrm{H}), 3.30(\mathrm{~s}, 3 \mathrm{H}) ;{ }^{13} \mathrm{C}$ NMR $(150$ $\left.\mathrm{MHz}, \mathrm{CDCl}_{3}\right) \delta: 164.29,162.61,158.03,134.73,133.69$, $132.91,131.15,130.71,130.24,129.95,128.85,128.41$, 125.86, 106.00, 51.51; HRMS (ESI) calcd for $\mathrm{C}_{17} \mathrm{H}_{14} \mathrm{ClO}_{4}$ $[\mathrm{M}+\mathrm{H}]^{+}$317.0575, found 317.0576.

(Z)-3-Methoxy-3-oxo-1-phenylprop-1-en-1-yl 4-(tertbutyl)benzoate (4l): White solid, $60.8 \mathrm{mg}, 90 \%$ yield. m.p. $149.1 \sim 149.8{ }^{\circ} \mathrm{C} ;{ }^{1} \mathrm{H}$ NMR $\left(600 \mathrm{MHz}, \mathrm{CDCl}_{3}\right) \delta: 8.18(\mathrm{~d}$, $J=7.8 \mathrm{~Hz}, 2 \mathrm{H}), 7.66$ (d, $J=7.8 \mathrm{~Hz}, 2 \mathrm{H}), 7.55$ (d, $J=7.8$ $\mathrm{Hz}, 2 \mathrm{H}), 7.43$ (d, $J=6.6 \mathrm{~Hz}, 1 \mathrm{H}), 7.40$ (t, $J=7.8 \mathrm{~Hz}, 2 \mathrm{H})$, $6.41(\mathrm{~s}, 1 \mathrm{H}), 3.68(\mathrm{~s}, 3 \mathrm{H}), 1.37(\mathrm{~s}, 9 \mathrm{H}) ;{ }^{13} \mathrm{C}$ NMR $(150$ $\left.\mathrm{MHz}, \mathrm{CDCl}_{3}\right) \delta: 164.46,163.80,158.38,157.44,133.41$, $130.97,130.24,128.79,126.17,125.95,125.68,106.04$, 51.49, 35.16, 31.06; HRMS (ESI) calcd for $\mathrm{C}_{21} \mathrm{H}_{23} \mathrm{O}_{4}[\mathrm{M}+$ $\mathrm{H}]^{+}$339.1591, found 339.1590.

4.3 General procedure for the intramolecular competition experiment

A $10 \mathrm{~mL}$ oven-dried reaction tube equipped with a magnetic stirrer bar was charged with bromoalkyne 1a $(0.20 \mathrm{mmol})$, BI-OAc $(0.20 \mathrm{mmol})$, silver carbonate (5 mol\%) and freshly distilled $\mathrm{CH}_{3} \mathrm{CN}(1.0 \mathrm{~mL})$ under air atmosphere. The reaction tube was placed in an oil bath and stirred at $90{ }^{\circ} \mathrm{C}$ for $6 \mathrm{~h}$. After the reaction was completed (detected by TLC), the mixture was cooled to room temperature, and extracted with ethyl acetate $(5 \mathrm{~mL} \times 3)$. The organic layers were combined, dried over anhydrous $\mathrm{MgSO}_{4}$, and concentrated under reduced pressure to yield the crude product, which was further purified by flash chromatography [silica gel, $V$ (petroleum ether) : $V$ (ethyl acetate) $=10: 1$ to $20: 1$ ], affording the desired product (Z)-2-bromo-1-phenylvinyl 2-iodobenzoate (5) as white solid (65.0 mg, 76\% yield). m.p. 61.4 62.8 ${ }^{\circ} \mathrm{C} ;{ }^{1} \mathrm{H}$ NMR $\left(600 \mathrm{MHz}, \mathrm{CDCl}_{3}\right) \delta: 8.26(\mathrm{dd}, J=7.8,1.8 \mathrm{~Hz}, 1 \mathrm{H}), 8.11$ $(\mathrm{d}, J=7.8 \mathrm{~Hz}, 1 \mathrm{H}), 7.54 \sim 7.52(\mathrm{~m}, 3 \mathrm{H}), 7.41 \sim 7.39(\mathrm{~m}$,
$3 \mathrm{H}), 7.28(\mathrm{t}, J=7.8 \mathrm{~Hz}, 1 \mathrm{H}), 6.70(\mathrm{~s}, 1 \mathrm{H}) ;{ }^{13} \mathrm{C} \mathrm{NMR}(150$ $\left.\mathrm{MHz}, \mathrm{CDCl}_{3}\right) \delta: 162.40,150.40,141.91,133.59,132.92$, $132.74,131.85,129.48,128.81,128.08,124.99$, 96.98, 95.11; HRMS (ESI) calcd for $\mathrm{C}_{15} \mathrm{H}_{10} \mathrm{BrINaO}_{2}[\mathrm{M}+\mathrm{Na}]^{+}$ 450.8801 , found 450.8801 .

\subsection{General procedure for the gram-scale synthesis of $3 a$}

A $25 \mathrm{~mL}$ oven-dried reaction tube equipped with a magnetic stirrer bar was charged with $\mathbf{1 a}(5.0 \mathrm{mmol})$, HIRs 2a $(5.00 \mathrm{mmol})$, silver carbonate $(2.5 \mathrm{~mol} \%)$ and freshly distilled $\mathrm{CH}_{3} \mathrm{CN}(10 \mathrm{~mL})$ under air atmosphere. The reaction tube was placed in an oil bath and stirred at $90{ }^{\circ} \mathrm{C}$ for $10 \mathrm{~h}$. After the reaction was completed, the mixture was cooled to room temperature, and extracted with ethyl acetate $(20 \mathrm{~mL} \times 3)$. The organic layers were combined, dried over anhydrous $\mathrm{MgSO}_{4}$, and concentrated under reduced pressure to yield the crude product, which was further purified by flash chromatography [silica gel, $V$ (petroleum ether) $: V$ (ethyl acetate $)=10: 1$ to $20: 1]$, affording the desired product 3a as pale yellow liquid (1.14 g, 72\%).

\subsection{General procedure for the late-stage transfor- mation}

Under nitrogen atmosphere, a $10 \mathrm{~mL}$ oven-dried reaction tube equipped with a magnetic stirrer bar was charged with 3i $(0.40 \mathrm{mmol})$, 1-phenylethyne (0.84 mmol), $\mathrm{Pd}(\mathrm{dppf})_{2} \mathrm{Cl}_{2}$ (1 mol \%), CuI (10 mol \%), triethylamine $(2.0 \mathrm{~mol})$ and anhydrous $N, N$-dimethylformamide (DMF, $1.0 \mathrm{~mL}$ ). The reaction tube was placed in an oil bath and stirred at $80{ }^{\circ} \mathrm{C}$ for $12 \mathrm{~h}$. After the reaction was completed, the mixture was cooled to room temperature, and extracted with ethyl acetate $(5 \mathrm{~mL} \times 3)$. The organic layers were combined, dried over anhydrous $\mathrm{MgSO}_{4}$, and concentrated under reduced pressure to yield the crude product, which was further purified by flash chromatography [silica gel, $V$ (petroleum ether) $: V($ ethyl acetate $)=9: 1$ ], affording the desired product (Z)-4-phenyl-1-(4-(phenylethynyl)phenyl)but-1-en-3-yn-1-yl 4-methylbenzoate (6) as white solid (122.7 mg, 70\%). m.p. $185.1 \sim 186.3{ }^{\circ} \mathrm{C} ;{ }^{1} \mathrm{H}$ NMR (600 MHz, $\left.\mathrm{CDCl}_{3}\right) \delta: 8.19(\mathrm{~d}, J=7.8 \mathrm{~Hz}, 2 \mathrm{H})$, $7.56 \sim 7.51(\mathrm{~m}, 6 \mathrm{H}), 7.36 \sim 7.34(\mathrm{~m}, 5 \mathrm{H}), 7.24 \sim 7.14(\mathrm{~m}$, $6 \mathrm{H}), 6.30(\mathrm{~s}, 1 \mathrm{H}), 2.49(\mathrm{~s}, 3 \mathrm{H}) ;{ }^{13} \mathrm{C} \mathrm{NMR}(150 \mathrm{MHz}$, $\left.\mathrm{CDCl}_{3}\right) \delta: 163.93,155.42,144.70,133.22,131.91,131.80$, $131.66,131.41,130.45,129.42,128.48,128.37,128.32$, $128.16,126.31,124.49,123.04,123.00,98.61,98.04$, 91.37, 89.05, 84.39, 21.80; HRMS (ESI) calcd for $\mathrm{C}_{32} \mathrm{H}_{23} \mathrm{O}_{2}[\mathrm{M}+\mathrm{H}]^{+}$439.1693, found 439.1693.

4.6 General procedure for the radical trapping experiments with TEMPO

A $10 \mathrm{~mL}$ oven-dried reaction tube equipped with a magnetic stirrer bar was charged with 1a $(0.20 \mathrm{mmol})$, (diacetoxyiodo)benzene (DIB, $0.20 \mathrm{mmol}$ ), silver carbonate $(2.5 \mathrm{~mol} \%)$, TEMPO $(0.40 \mathrm{mmol})$ and freshly distilled $\mathrm{CH}_{3} \mathrm{CN}(1.0 \mathrm{~mL})$ under air atmosphere. The reaction tube was placed in an oil bath and stirred at $90{ }^{\circ} \mathrm{C}$ for $6 \mathrm{~h}$. After that, the reaction mixture was cooled to room tem- 
perature, and extracted with ethyl acetate $(5 \mathrm{~mL} \times 3)$. The organic layers were combined, dried over anhydrous $\mathrm{MgSO}_{4}$, and concentrated under reduced pressure to yield the crude product, which was further purified by flash chromatography [silica gel, $V$ (petroleum ether) : $V$ (ethyl acetate) $=10: 1$ to $20: 1$ ], affording the desired product $4 \mathbf{e}$ in $32 \%$ yield. Therefore, a radical pathway can be ruled out for this silver-catalyzed system.

\subsection{General procedure for deuterium-labeled ex- periments}

A $10 \mathrm{~mL}$ oven-dried reaction tube equipped with a magnetic stirrer bar was charged with 1a $(0.20 \mathrm{mmol})$, (diacetoxyiodo)benzene (DIB, $0.20 \mathrm{mmol}$ ), silver carbonate $(2.5 \mathrm{~mol} \%), \mathrm{D}_{2} \mathrm{O}(0.20 \mathrm{mmol})$ and freshly distilled $\mathrm{CH}_{3} \mathrm{CN}(1.0 \mathrm{~mL})$ under $\mathrm{N}_{2}$ atmosphere. The reaction tube was placed in an oil bath and stirred at $90{ }^{\circ} \mathrm{C}$ for $6 \mathrm{~h}$. After the reaction was completed, the mixture was cooled to room temperature, and extracted with ethyl acetate $(5 \mathrm{~mL}$ $\times 3$ ). The organic layers were combined, dried over anhydrous $\mathrm{MgSO}_{4}$, and concentrated under reduced pressure to yield the crude product, which was further purified by flash chromatography [silica gel, $V$ (petroleum ether) : $V($ ethyl acetate $)=10: 1$ to $20: 1$ ], affording the desired product $4 \mathbf{e} /[\mathrm{D}]-\mathbf{4 e}$ (ca. $29 \%$ total yield). The ratio of mixture ([D]-4e/4e $=1.86)$ was determined by ${ }^{1} \mathrm{H}$ NMR analysis of the obtained mixture, which indicates the pronation step was involved therein.

Supporting Information ${ }^{1} \mathrm{H}$ NMR and ${ }^{13} \mathrm{C}$ NMR spectra of new compounds $3 \sim \mathbf{6}$. The Supporting Information is available free of charge via the Internet at http://siocjournal.cn/.

\section{References}

[1] (a) Haines, A. H. Methods for the Oxidation of Organic Compounds. Alkanes, Alkenes, Alkynes, and Arenes, Academic Press, New York, 1985.

(b) Meldal, M.; Tornoe, C. W. Chem. Rev. 2008, 108, 2952.

(c) Hein, J. E.; Fokin, V. V. Chem. Soc. Rev. 2010, 39, 1302.

(d) Gilmore, K.; Alabugin, I. V. Chem. Rev. 2011, 111, 6513.

[2] (a) Wu, W.; Jiang, H. Acc. Chem. Res. 2014, 47, 2483.

(b) Fang, G.; Bi, X. Chem. Soc. Rev. 2015, 44, 8124.

(c) Zhang, F.; Peng, X.; Ma, J. Chin. J. Org. Chem. 2019, 39, 109 (in Chinese).

(张发光, 彭星, 马军安, 有机化学, 2019, 39, 109.)

(d) Cheng, Z.; Guo, J.; Lu, Z. Chem.Commun.2020, 56, 2229.

(e) Ackermann, L. Acc. Chem. Res. 2020, 53, 84.

[3] (a) Al-awar, R. S.; Joseph, S. P.; Comins, D. L. J. Org. Chem. 1993, 58,7732 .

(b) Kamei, K.; Maeda, N.; Tatsuoka, T. Tetrahedron Lett. 2005, 46, 229.

(c) Spaggiari, A.; Vaccari, D.; Davoli, P.; Torre, G.; Prati, F. J. Org.

Chem. 2007, 72, 2216.

(d) Su, W.; Jin, C. Org. Lett. 2007, 9, 993.

(e) Iwai, T.; Fujihara, T.; Terao, J.; Tsuji, Y. J. Am. Chem. Soc. 2012, 134, 1268 .

(f) Wu, X.-F.; Bezier, D.; Darcel, C. Adv. Synth. Catal. 2009, 351,
367.

[4] (a) Chen, Z.; Li, J.; Jiang, H.; Zhu, S.; Li, Y.; Qi, C. Org. Lett. 2010 , 12,3262 .

(b) Chen, Z.; Jiang, H.; Li, Y.; Qi, C. Chem. Commun. 2010, 46, 8049.

(c) Jiang, G.; Zhu, C.; Li, J.; Wu, W.; Jiang, H. Adv. Synth. Catal. 2017, 359, 1208.

[5] Mo, D.-L.; Dai, L.-X.; Hou, X.-L. Tetrahedron Lett. 2009, 50 , 5578.

[6] (a) Wang, S.; Li, P.; Yu, L.; Wang, L. Org. Lett. 2011, 13, 5968. (b) Hong, X.; Ma, F.; Zha, D.; Li, H. Asian J. Org. Chem. 2018, 7, 2552.

[7] Zeng, X.; Liu, S.; Shi, Z.; Xu, B. Org. Lett. 2016, $18,4770$.

[8] Lu, X.; Zhu, G.; Ma, S. Tetrahedron Lett. 1992, 33, 7205.

[9] (a) Zhdankin, V. V.; Stang, P. J. Chem. Rev. 2008, 108, 5299.

(b) Charpentier, J.; Früh, N.; Togni, A. Chem. Rev. 2015, 115, 650.

(c) Yoshimura, A.; Zhdankin, V. V. Chem. Rev. 2016, 116, 3328.

(d) Duan, Y.; Jiang, S.; Han, Y.; Sun, B.; Zhang, C. Chin. J. Org. Chem. 2016, 36, 1973 (in Chinese).

(段亚南, 姜山, 韩永超, 孙博, 张弛, 有机化学, 2016, 36, 1973.) (e) Zhang, H.; Tang, R.; Shi, X.; Xie, L.; Wu, J. Chin. J. Org. Chem. 2019, 39, 1837 (in Chinese).

(张怀远, 唐蓉萍, 石星丽, 颕林, 伍家卫, 有机化学, 2019, 39, 1837.)

(f) Wang, X.; Studer, A. Acc. Chem. Res. 2017, 50, 1712.

For recent examples, see: (g) Chen, Q.; Yang, Y.; Wang, X.; Zhang, Q.; Li, D. Chin. J. Org. Chem. 2020, 40, 454 (in Chinese).

(陈倩雯, 杨耀成, 王霞, 张谦, 李栋, 有机化学, 2020, 40, 454.)

(h) Gao, P.; Fan, M.; Bai, Z.; Wei, Y. Chin. J. Chem. 2015, 33, 479.

[10] (a) Frei, R.; Wodrich, M. D.; Hari, D.; Borin, P.; Chauvier, C.; Waser, J. J. Am. Chem. Soc. 2014, 136, 16563.

(b) Zhang, J.; Szabo, K, J.; Himo, F. ACS Catal. 2017, 7, 1093.

(c) Wang, S.; Gu, Q.; You, S. J. Org. Chem. 2017, 82, 11829.

(d) Xia, H.-D.; Zhang, Y.-D.; Wang, Y.-H.; Zhang, C. Org. Lett. 2018, 20, 4052.

(e) Li, J.; Liu, Z.; Wu, S.; Chen, Y. Org. Lett. 2019, 21, 2077.

(f) Li, G.-X.; Hu, X.; He, G.; Chen, G. Chem. Sci. 2019, 10, 688

(g) Lan, T.; Zhang, Y.; Liu, W.; Xi, C.; Chen, C. Chin. J. Org Chem. 2019, 39, 2166 (in Chinese).

(兰天否, 张越, 刘伟, 席婵娟, 陈超, 有机化学, 2019, 39, 2166.)

(h) Liu, Q.-R.; Pan, C.-X.; Ma, X.-P.; Mo, D.-L.; Su, G.-F. J. Org. Chem. 2015, 80, 6496.

(i) Ma, X.-P.; Li, K.; Wu, S.-Y.; Liang, C.; Su, G.-F.; Mo, D.-L. Green Chem. 2017, 19, 5761.

[11] (a) Tan, H.; Li, H.; Ji, W.; Wang, L. Angew. Chem., Int. Ed. 2015 54,8374 .

(b) Zha, D.; Li, H.; Li, S.; Wang, L. Adv. Synth. Catal. 2017, 359, 467.

(c) Wang, L.; Li, H.; Wang, L. Org. Lett. 2018, 20, 1663.

(d) Yang, S.; Li, H.; Li, P.; Yang, J.; Wang, L. Org. Biomol. Chem 2020, 18,715 .

[12] Deng's group reported a reaction of terminal alkynes with DIB using AgOAc as a catalyst, but which only forms $\alpha$-acetoxy ketones as sole products. Under Deng's conditions, this stereoselective addition reaction of functionalized alkynes with hypervalent iodine(III) reagents does not give the desired product (Entry 2, Table 1). Based on these experiment results, a different pathway for this silver-catalyzed addition is proposed in Scheme 5.

[13] (a) Yamada, W.; Sugawara, Y.; Cheng, H. M.; Ikeno, T.; Yamada, T. Eur. J. Org. Chem. 2007, 16, 2604.

(b) Deng, G.; Luo, J. Tetrahedron 2013, 69, 5937.

[14] Jiang, G.; Li, J. X.; Zhu, C.; Wu, W.; Jiang, H. Org. Lett. 2017, 19, 4440 . 